\title{
The effect of super-oxidized water on the tissues of uterus and ovary: An experimental rat study
}

\author{
Abbas Aras ${ }^{1}$, Erbil Karaman ${ }^{2 \star}$, Numan Çim ${ }^{2}$, Serkan Yıldırım ${ }^{3}$, Remzi Kızıltan ${ }^{1}$, Özkan Yılmaz ${ }^{1}$ \\ ${ }^{1}$ Department of General Surgery, Medical Faculty, Yuzuncu Yil University, Van, Turkey \\ ${ }^{2}$ Department of Obstetric and Gynecology, Medical Faculty, Yuzuncu Yil University, Van, Turkey \\ ${ }^{3}$ Department of Pathology, Veterinary Faculty, Ataturk University, Erzurum, Turkey
}

\begin{abstract}
Super-oxidized solutions are known to be potent disinfectants for external surfaces and also for wound care. There are limited data about the use of superoxidized water in the intraperitoneal organs. The aim of the present study was to evalaute its effect on the uterus and ovary when applied via intraperitoneal infusion in a rat model.

Thirty Wistar-Albino rats weighing 250-300 g were randomly divided into three groups (10 rats/group). Group1(control group) rats received single dose of $10 \mathrm{mg} / \mathrm{kg}$ saline solution intraperitoneally. Group 2(single dose group) rats received single dose of $10 \mathrm{mg} / \mathrm{kg}$ pH-neutral SOW intraperitoneally. Group 3(multiple doses group) rats received multiple doses of $10 \mathrm{mg} / \mathrm{kg} \mathrm{pH}$-neutral SOW intraperitoneall at first, third and fifth days. All animals were sacrificed at one week after infusion. The macro- and microscopic histopathological examinations were performed for each rat.

All rats remained healthy follow up of one week. The macroscopic examinations of the three groups showed no significant differences. No toxicity findings were found in three groups. The microscopic examinations revealed active endometial glandular structures in uterus and functional follicules at different stages of maturation in ovary. There were no significant differences with regards to the microscopic findings between three groups.

Intraperitoneal infusion of $\mathrm{pH}$-neutral SOW does not result in any significant toxicity and complications on the tissues of uterus and ovary.
\end{abstract}

Key Words: Super-oxidized water, rat study, uterus, ovary, toxicity

\section{Introduction}

Super-oxidized water (SOW) is a relatively new formulated $\mathrm{pH}$-neutral solution that has a potent disinfectant activity (1). SOW does not cause harmful effect on the human tissues and used for various conditions like wound, skin infections, ulcers and diabetic foot $(2,3)$. The other forms of this formulation were not neutral $\mathrm{pH}$ and has a strong acid aqueous ingredient and theoretically may lead to toxic and negative effect on the tissue and subcellular level (4). SOW is generated by exerting electrical current on salty water and conduct electrochemically in aqueous solutions from water and sodium chloride. Water is reduced to oxygen, ozone and other reactive species however, the major ingredient formed during this process is hypochlorite and hypochlorous. On marketing, there are a variety of compounds that have different concentrations and $\mathrm{pH}$ values (5).

On literature search, it was reported and confirmed by several studies that SOW has a potent antimicrobial activity including viruses and bacteria (6). Although SOW is used mainly for hospital wall's surfaces and metal health's equipment, many published data investigated its effect on external surface of body such as in diabetic foot ulcers, burn wounds and skin infections (7). However, there are limited data about the use of SOW in intraabdominal organs or within the integrity of body system. Two studies investigated the effect of super-oxidized solutions on the perforated appendicitis with intraabdominal use and found beneficial effects. However, these molecules were in strong acid component. The tuba-ovarian abscess and pelvic peritonitis are common complications of pelvic inflammatory disease. During the surgery of these conditions, abscess drainage is maintained with addition of normal saline irrigation of peritoneal cavity which is in fact has no microbicidal activity. Therefore, it will be rational to use a potent $\mathrm{pH}$ neutral and non-toxic agent like SOW during these operations. As far as we know, there is no study that evaluated the effect, toxicity and complications of SOW on uterine and ovary tissue during direct intraabdominal infusion.

Aim of this study is to conduct an experimental rat study to investigate the macro and microscopic findings in uterine and ovary tissues following the 
intraperitoneal infusion of SOW solution in rats and also to determine any toxic effect of SOW.

\section{Material and methods}

This experimental study was conducted in animal research laboratory of a University Hospital. The study was approved by the Ethics committee for Animal Experiments of that University. The reference number of was 19 at 26/06/2015. Thirty Wistar-Albino rats (weight 250-300 gr) were used as a model in accordance with Declaration of Helsinki. All the animals were fed with ad libitum with food approved by Standards Institute. The rats were randomly divided equally into three groups (10 rats in each group).

Experimental groups: All rats were administered by intraperitoneal infusion of SOW (Crystalin, Wound Care, NPS, İstanbul, TURKEY). The procedure was performed by one of our authors who has registration and certificate for animal research Project (A.A.). The experimental groups were divided into three groups as follows:

Group 1 (control group, intraperitoneal saline): An intraperitoneal single dose of $10 \mathrm{cc} / \mathrm{kg} 0.9$ (normal saline) was injected into the pelvic peritoneal cavity using 22-French syringes.

Group 2 (single dose intraperitoneal SOW group): Rats received a single dose of $10 \mathrm{cc} / \mathrm{kg}$ SOW via injection into the pelvic peritoneal cavity using 22French syringes.

Group 3 (Multiple dose intraperitoneal SOW group): The intraperitoneal multiple dose of 10 $\mathrm{cc} / \mathrm{kg}$ SOW was administered on the first, third and fifth day with 22-French syringes. After injections the rats were returned to Standard vivarium care.
After one week of follow up, the rats were sacrificed under $75 \mathrm{mg} / \mathrm{kg}$ Ketamin anesthesia. Each on its back on the operation table and pleuro-peritoneal cavity was opened. The pelvic peritoneal cavity, uterus, bilateral fallopian tubes and bilateral ovaries were examined macroscopically. Any macroscopic changes including adhesions, ulcerations or notable differences were noted by the experienced pathologist who is one of the authors (S.Y.) and blinded to the three groups. The tissue specimens of uterus including endometrium, myometrium and perimetrium and ovarian tissue samples including cortex and medulla were obtained. Following dehydration and paraffinization, the tissues were cut into sections (5 $\mu \mathrm{m}$ thick) and stained with hematoxylin and eosin. Samples were examined under a light microscope. Histopathologic examinations were done by the pathologist who was blinded to the groups.

\section{Results}

The rats were followed up for one week and all the animals survived until at the end of experimental study after injection of SOW. No rats showed any surgical site reaction regarding injection of SOW on abdominal cavity including infection or allergy. The macroscopic examination of three groups showed no changes in the pelvic, uterus fallopian tubes, and the other intraabdominal organs (Figure 1). No pathologic findings were noted on macroscopic view. On microscopic examination, the three groups' uterine were examined and showed no differences between groups (Figure 2). There was no toxic or structural deterioration within endometrial glands and myometrial tissue.

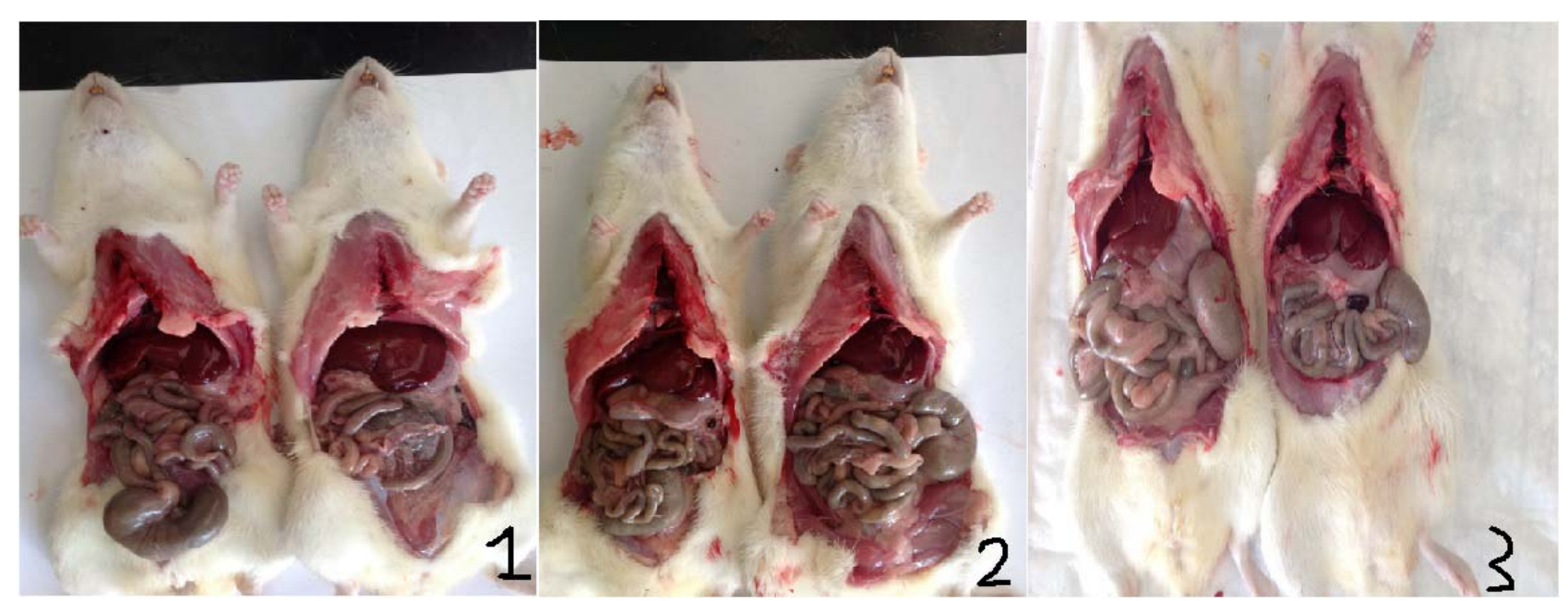

Fig. 1. Shows the macroscopic findings of the group 1,2 and 3 as presented. 


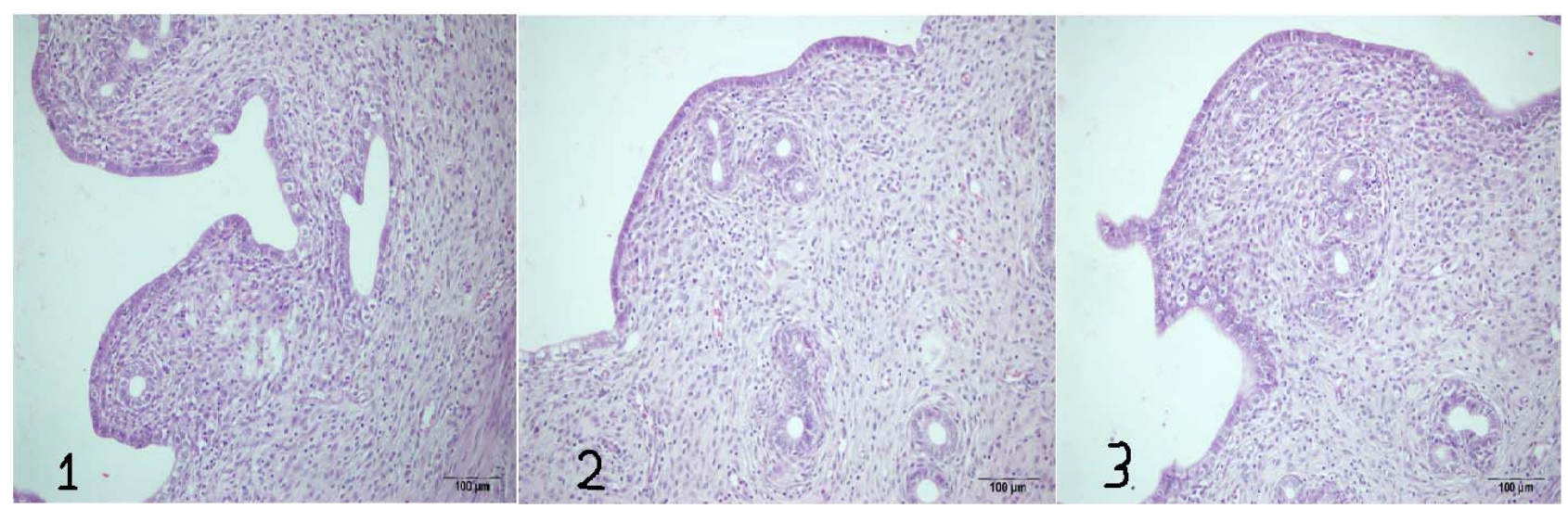

Fig. 2. Shows the microscopic examination of the three groups that presents the active glandular structures in endometrium of the rats.

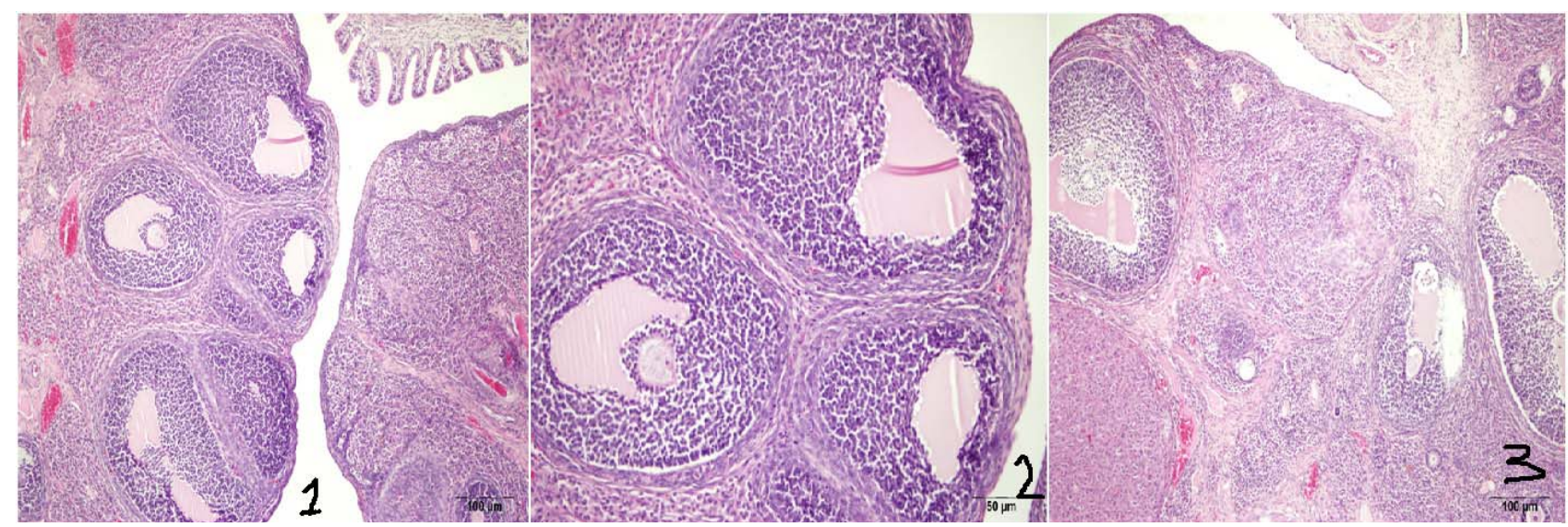

Fig. 3. Shows the microscopic examination of the three groups that presents the functional in ovarian cortex at different stages of maturation.

The microscopic of ovarium revealed no changes on ovarian surfaces in all three experimental groups. No histologic differences were noted between groups (Figure 3). The three groups were found to have primordial, primary, secondary, graafian and corpus luteum in which are rational findings that show the normal integrity of tissue.

On toxicologic examination, the presence of active endometrial glandular within uterus and within ovaries in each three groups identified no toxic effect of SOW administration for single and multiple repeated dosages.

\section{Discussion}

Super-oxidized water (SOW), used in our experimental study, is a $\mathrm{pH}$-neutral solution and has a potent antimicrobial efficacy. This solution being used for many conditions including nonorganic and organic surfaces. Currently, it is used in hospital equipments for its beneficial effect on microorganisms as a disinfectant (8). However, the first used super-oxidized solutions had strong acid aqueous content and had potential role of become toxic and hazardous to human surfaces and tissues. The new generation SOW solutions have a $\mathrm{pH}$-neutral with a longer shelf-life $(>12$ months) than the former super-oxide solutions. These new generation SOW are intended for the topical treatment of infective chronic and acute wounds like diabetic ulcers. Although there are several studies that evaluated the external use of these products in human body, there is very limited data about the use of $\mathrm{pH}$-neutral SOW in intraperitoneal organs. So we aimed to conduct such an experimental rat study to investigate the complications and toxic effects of the intraperitoneal infusion of SOW on uterine and ovarian tissues. Our study findings showed that the use of SOW intraperitoneally does not has any toxicity effects and complications on uterine and ovarian tissues. And, there were no significant differences between the use of single versus multiple repeated doses of SOW with regards to the tissue reactions.

The super-oxidized solutions have been found to be very efficient molecules that can fight against different types of microorganisms including spore-

East J Med Volume:22, Number:1, January-March/2017 
forming bacteria, viruses and fungi (9). Park et al (10) studied on the evaluation of liquid- and fogbased application of hypochlorous acid solution for surface inactivation of human noroviruses which are the most frequent causes of gastroenteritis outbreaks with surface mediated transfer via contact. They reported that Hypochlorous acid is likely to be effective in disinfecting in common settings to reduce norovirus exposures. Another $\mathrm{pH}$-neutral superoxidized water solution called microcyn investigated and found to have a wide antimicrobial spectrum with major advantages over acidic SOWs, including neutral $\mathrm{pH}$, lower free active chlorine (51-85 ppm) and long shelf life (1 year) (11). In a previous experimental study that used a rat burn model investigated the effect of electrolyzed oxidized water on pseudomonas aeruginosa infection (12). The former study reported that irrigation and disinfection with electrolyzed oxidized water may become useful in preventing burn-wound sepsis (12).

In literature search, we found an interesting study by Kubota et al (6) who studied on the efficacy and safety of strong acid electrolyzed water for peritoneal lavage to prevent surgical site infection in patients with perforated appendicitis. They found that peritoneal lavage and wound washing with strong acid electrolyzed water have no adverse effects and are effective for preventing surgical site infection. However, the previous study used a strong acid solution, not a $\mathrm{pH}$-neutral oxidized water in which evaluated in our study. As far as we know from literature, there is no study that investigated the intraperitoneal use of SOW. The peritoneal infection that is caused by pelvic inflammatory disease may involve the uterus, and ovaries in which may be resulted in tubo-ovarian abscess formation. The routine gynecology practice uses normal saline irrigation during the surgical treatment of this intraabdominal abscess formation. The saline, in fact, has no microbicidal activity. The use of a non-toxic and disinfectant agent would be very helpful during these procedures. Before the application of a SOW intraperitoneally in human body, we thought that it should be investigated in an experimental study in order to find whether it has any toxic effect or complications on uterus and ovaries or not. Our study showed that it has no toxic effect on uterus and ovarian tissues.

The normal functioning uterine glandular structures and absence of atretic follicles and necrosis of oocytes and granulosa cells are the indirect signs of non-toxic effect of SOW infused intraperitoneally (13). In our study, the ovarian tissue showed functional at different stages of maturation and the uterine glandular changes were not different between the three groups. In normal situation, the chemical compounds that have contacted with human tissues have an accumulation effect. This was investigated in our study and the multiple of SOW that applied on the uterine and ovarian tissues showed no difference in macro-and microscopic toxic reactions on these tissues.

This study has several limitations. First, no longterm follow up of greater than one week to observe the toxic reaction or complications has been done. However, we think that this disinfectant solution shows its effect immediately when it comes contact with the tissue and one week will be enough to see the toxic effect. Second, we focused on uterine and ovarian tissue complications after infusion of SOW. We did not assess the other systemic changes that will be resulted with hormonal changes. On the other hand, the strength of our study was that all histopathologic examinations were performed by the same experienced pathologist.

In conclusion, we conducted an experimental rat study that is performed with intraperitoneal infusion of $\mathrm{pH}$-neutral new generation superoxidized water solution and found no histopathological evidence of adverse toxic reaction and complication on the uterine and ovarian tissues. Our study results showed that SOW can be used in intraperitoneal abscess formation that involved the ovary without resulting significant toxic reactions and complications.

Conflict of interest: The authors declare that they have no conflict of interest.

\section{References}

1. Hadi SF, Khaliq $\mathrm{T}$, Bilal $\mathrm{N}$, et al. Treating infected diabetic wounds with superoxidized water as anti-septic agent: a preliminary experience. J Coll Physicians Surg Pak 2007; 17(12): 740-743.

2. Miranda-Altamirano A. Reducing bacterial infectious complications from burn wounds. A look at the use of Oculus Microcyn60 to treat wounds in Mexico. Wounds Jan 2006: 17-9 (Suppl).

3. Yahagi N, Kono M, Kithara M, et al. Effect of electrolyzed water on wound healing. Artif Organs 2000; 24: 984-987. 
4. Imatanaka N, Yamasaki $\mathrm{K}$, Shiraishi $\mathrm{K}$, et al. Single dose and 28-day repeated dose oral toxicity studies of superoxidized water in rats. Pharmacometrics I994: 48: 159-171.

5. Gunaydin M, Esen S, Karadag A, et al. In vitro antimicrobial activity of Medilox ${ }^{\circledR}$ superoxidized water. Ann Clin Microbiol Antimicrob. 2014; 13: 29.

6. Kubota A, Goda T, Tsuru T, et al. Efficacy and safety of strong acid electrolyzed water for peritoneal lavage to prevent surgical site infection in patients with perforated appendicitis. Surg Today 2015; 45: 876-879.

7. Tanaka H, Hirakata Y, Kaku M, et al. Antimicrobial activity of superoxidized water. J Hosp Infect 1996; 34: 43-49.

8. Tanaka N, Tanaka N, Fujisawa T, et al. The use of electrolyzed solutions for the cleaning and disinfecting of dialyzers. Artif Organs 2000; 24: 921-928.

9. Shimizu Y, Sugawara H. Virucidal and bactericidal effects of electrolyzed oxidizing water: comparison of disinfectant effect with electrolyzed oxidizing water and hypochlorous acid. Jpn J Oral Biol 1996; 38: 564-571.

10. Park GW, Boston DM, Kase JA, Sampson MN, Sobsey MD. Evaluation of liquid- and fog-based application of Sterilox hypochlorous acid solution for surface inactivation of human norovirus. Appl Environ Microbiol 2007; 73: 4463-4468.

11. Landa-Solis C, González-Espinosa D, GuzmánSoriano B, et al. Microcyn: a novel superoxidized water with neutral $\mathrm{pH}$ and disinfectant activity. J Hosp Infect 2005; 61: 291-299.

12. Nakae H, Inaba H. Effectiveness of electrolyzed oxidized water irrigation in a burn-wound infection model. J Trauma 2000; 49: 511-514.

13. Sun J, Zhang X, Cao Y, et al. Ovarian Toxicity in Female Rats after Oral Administration of Melamine or Melamine and Cyanuric Acid. PLoS One 2016; 11: e0149063. 